\title{
Material-independent crack arrest statistics
}

\author{
Yann Charles, ${ }^{\text {a }}$ Damien Vandembroucq, ${ }^{\mathrm{b}}$ François Hild ${ }^{\mathrm{a}}$ \\ and Stéphane Roux b,1 \\ ${ }^{\mathrm{a}} L M T-C a c h a n$ \\ ENS de Cachan / CNRS-UMR 8535 / Université Paris 6 \\ 61 avenue du Président Wilson, F-94235 Cachan Cedex, France. \\ ${ }^{\mathrm{b}}$ Surface du Verre et Interfaces, \\ Unité Mixte de Recherche CNRS/Saint-Gobain \\ 39 quai Lucien Lefranc, F-93303 Aubervilliers Cedex, France.
}

\begin{abstract}
The propagation of (planar) cracks in a heterogeneous brittle material characterized by a random field of toughness is considered, taking into account explicitly the effect of the crack front roughness on the local stress intensity factor. In the socalled strong-pinning regime, the onset of crack propagation appears to map onto a second-order phase transition characterized by universal critical exponents which are independent of the local characteristics of the medium. Propagation over large distances can be described by using a simple one-dimensional description, with a correlation length and an effective macroscopic toughness distribution that scale in a non-trivial fashion with the crack front length. As an application of the above concepts, the arrest of indentation cracks is addressed, and the analytical expression for the statistical distribution of the crack radius at arrest is derived. The analysis
\end{abstract}


of indentation crack radii on alumina is shown to obey the predicted algebraic expression for the radius distribution and its dependence on the indentation load.

Keywords: A. Crack propagation and arrest; A. Indentation and hardness; B. Inhomogeneous material; C. Probability and statistics; Brittle material.

1 to whom correspondence should be addressed.

e-mail: stephane.roux@saint-gobain.com 


\section{Introduction}

Brittle materials, such as ceramics and glasses, are known to be extremely sensitive to bulk and surface defects, from which cracks can be initiated eventually leading to failure. This extreme sensitivity calls for a statistical analysis of crack initiation, which has been extensively developed following the pioneering work of Weibull (Weibull, 1939). In this "weakest-link" approach (Freudenthal, 1968), the analysis is focused on the initiation stage, implicitly assuming that the propagation stage is obtained systematically over unlimited distances, due to the lower loading needed for this stage as compared to the inception of propagation.

However, in some cases, even though cracks are initiated, they will not propagate over large distances, and their presence may still be acceptable in service condition of a given structure. For example, in ceramic / metal assemblies, residual stresses caused by the coefficient of thermal expansion mismatch may prevent cracks to traverse the brittle part so that a weakest link hypothesis does not apply (Charles and Hild, 2002). In brittle-matrix composites, crack arrest is also observed due to the bridging forces induced by the fibers (Evans, 1990). Other examples of such confined cracks are those produced by indentation (Lawn, 1993). In the latter case, the stress field has a rapid decay with distance from the indentation point, and hence a crack that can easily be nucleated may stop shortly after initiation.

The mechanical treatment of crack arrest in a brittle material is standard: namely, Linear Elastic Fracture Mechanics generally holds without much restriction, and hence, a crack is expected to be arrested as soon as the stress intensity factor $K$ becomes smaller than the toughness $K_{c}$. The purpose of 
the present paper is to investigate the effect of a statistical distribution of toughness. A statistical model is considered for the steady propagation of an extended planar crack front through a random landscape of toughness. This situation is shown to give rise to a genuine phase transition at the onset of propagation. The recognition of this critical character opens to numerous interesting properties, not only concerning the complexity of the spatiotemporal development of crack propagation, but also and more importantly on the statistical distribution of the global stress intensity factor characterizing the entire front. In particular, universality justifies the independence of some properties on the details of the random toughness field. At a larger scale, the problem of crack arrest is considered in a non-uniform loading geometry. In this situation, some of the features revealed by the previous analysis can be used. As an application of the above concepts, the case of indentation cracks is considered since they are naturally arrested because of the decaying stress intensity factor. The semi-circular geometry of the crack front will allow for an explicit analysis of the statistical distribution of crack arrest distance. The results are applied on indentation of sintered alumina samples. Four different statistical records of indentation crack length for different loads on the same material are tested against the theoretical predictions.

\section{Crack pinning}

The three-dimensional problem where the crack front may be pinned at different locations leads to a complex problem, because elastic interactions affect the local stress intensity factor along the crack front. As a result, the local stress intensity factor depends on the overall front geometry in a non-local manner (Bower and Ortiz, 1991, 1993). Curtin (Curtin, 
1997, 1998) performed a simplified analysis of a similar problem. A modelling of planar crack propagation in a random environment was also proposed by Schmittbuhl et al. (Schmittbuhl et al., 1995a), Ramanathan and Fisher (Ramanathan and Fisher, 1998) and Bouchaud et al. (Bouchaud et al., 1993) that revealed a complex spatio-temporal organization of the propagation, and scale-invariant crack front morphology. These studies show that the macroscopic toughness is not simply given by the average toughness and that tougher elements impede crack growth in a lesser extend than with a strongest link assumption due to the stress intensity factor enhancement caused by crack front curvature. One of the most striking predictions of these approaches is the scale invariant nature of the crack front or crack surface roughness, which is qualitatively (unfortunately not quantitatively) in agreement with a large body of experimental analysis, a review of which is presented in (Bouchaud, 1997).

This problem, at this level of generality, is extremely complex, and simplifying assumptions are proposed to address it. First, the crack remains planar in the $(x, y)$-plane where the mean crack front is aligned along the $x$-direction, whereas the $y$-axis is the direction of crack propagation (Fig. 1). Second, the distribution of local toughness is sufficiently narrow so that a first-order perturbative analysis can be used to characterize the coupling of the local stress intensity factor $K(x)$ to the overall front morphology $h(x)$. Third, the elastic properties of the medium can be considered as homogeneous. Those assumptions allow one to use a result derived by Gao and Rice (Gao and Rice, 1989) that relates the local stress intensity factor $K(x, h(x))$ along the crack front to the crack front shape $h(x)$, and the reference stress intensity factor, $K_{0}$, which would result from the same loading with a straight crack front at the 
same mean position $h_{0}=\langle h\rangle_{x}$

$$
K(x, h(x))=K_{0}\left(1+\frac{1}{\pi} \int \frac{h(x)-h\left(x^{\prime}\right)}{\left(x-x^{\prime}\right)^{2}} \mathrm{~d} x^{\prime}\right) .
$$

As discussed in (Roux et al., 2003), those assumptions are not yet sufficient to specify entirely the problem at hand. The correlation function of the toughness pattern comes into play to discriminate two regimes:

- for large correlation lengths along the propagation direction, a weak-pinning regime is obtained, where the crack front is only mildly affected by the disorder and hence the crack advance at each point along the front is continuous as the mean position of the crack front $\langle h\rangle_{x}$ moves;

- for small correlation lengths along the propagation direction, the front undergoes a series of jumps from one stable position to the next. This regime, referred to as "strong-pinning," is the most interesting in the sense that the effective toughness is expected to depart from the arithmetic average of the local toughness (in contrast to the weak pinning regime).

In the following, only the latter regime is considered with a specific design of the local toughness distribution so that strong-pinning will be guaranteed.

\section{Propagation model}

Let us consider a planar crack front extending over the $x$-axis and propagating along the $y$-direction. The geometry of the front is described by $h(x, t)$, the $y$-coordinate of the front at an abscissa $x$ and time $t$. The model describes the propagation of the crack from one pinned configuration to the next. A time coordinate is introduced to label the sequence of crack front geometries. However, as explained below, no physical time-dependent propagation rule is 
considered, and hence this label is used as a convenient way of characterizing the mean crack advance. The front is discretized along the $x$-axis with a constant interval whose physical meaning can be interpreted as the correlation length of the toughness along the $x$-direction. The assumption that the crack front can be represented as a single-valued function of $x$ is a necessary assumption for the use of the perturbative result (1). The latter imposes the slope of the front to be small compared to unity (Gao and Rice, 1989). This in turn has consequences on the amplitude of the local toughness distribution, which has to be small compared to the mean value, $\bar{K}_{c}$. This quantity is the small parameter used in all subsequent expansions.

Under an external load that would produce a stress intensity factor $K_{0}(t)$ for a straight front, Eq. (1) allows one to estimate the local stress intensity factor, $K(x, t)$. The local toughness along the crack front $K_{c}(x, h(x, t))$ is assumed to be distributed without spatial correlation from a given distribution $p\left(K_{c}\right)$. To make the problem non-dimensional, the scaled stress intensity factor is defined as $k=\left(K-\bar{K}_{c}\right) / \bar{K}_{c}$, and the local dimensionless toughness $k_{c}=$ $\left(K_{c}-\bar{K}_{c}\right) / \bar{K}_{c}$. The local stress intensity factor can thus be written, as a first order approximation,

$$
k(x, t)=k_{0}(t)+k_{e l}(x, t)
$$

with

$$
k_{e l}(x, t)=\frac{1}{\pi} \int \frac{h(x, t)-h\left(x^{\prime}, t\right)}{\left(x-x^{\prime}\right)^{2}} \mathrm{~d} x^{\prime}
$$

One can compute the maximum level for which the crack would not propagate, $k_{0}(t)<k_{c}(x, h(x, t))-k_{e l}(x, t)$, for all $x$ along the front. Thus, the onset of crack propagation is given by a level such that the scaled global stress intensity 
factor assumes the value

$$
\kappa(t)=\min _{x}\left[k_{c}(x, h(x, t))-k_{e l}(x, t)\right]
$$

For such a level, at one point $x_{a c t}(t)$ along the front the stress intensity factor matches exactly the local toughness. Let us now introduce the way the crack propagation is modeled. At the point $x_{a c t}(t)$, the crack can overcome the local toughness and advance. It will directly jump to a new obstacle $h\left(x_{a c t}(t), t+1\right)=h\left(x_{a c t}(t), t\right)+\delta h$, where $\delta h$ can be either a constant length, or a random one. It characterizes the correlation along the propagation direction, and there lies the "strong-pinning" nature of the present model. At this new position, another local toughness is encountered, which is uncorrelated with the previous ones. All other sites are supposed to remain pinned during this elementary move of the crack front. The new front geometry and local toughness distribution is treated the same way. This means that the external loading is adjusted in time so that one constantly remains at the onset of propagation given by Eq. (4), irrespective of the variation of $\kappa(t)$. The physical time needed to achieve the elementary move of the crack front is not introduced here, and hence time appears here as a discrete label given to the different crack configurations. However, when inertia effects are negligible, one can reconstruct from the above signal $\kappa(t)$ what would be the evolution of the system under any specific history of loading in a quasi-static regime. In particular, for a subcritical but constant loading, $k_{0}$, starting from any configuration, one can compute the expected crack advance before reaching a pinned configuration for which $\kappa(t)>k_{0}$ for the first time.

For a crack front having a finite extent (with free or periodic boundary conditions), one can follow numerically the change of a crack front for arbitrarily long times, and record statistical information concerning either the crack front 
geometry, the activity of the front with time $\left[i . e .\right.$, position of $\left.x_{a c t}(t)\right]$, or the loading $\kappa(t)$. The latter signal is of special interest since it provides an estimate of the (reduced) critical loading $\kappa^{*}$ for which an unlimited propagation would be obtained

$$
\kappa^{*}=\max _{t}[\kappa(t)]
$$

and thus the latter corresponds to the macroscopic effective toughness of the medium, for extended propagation, while $\kappa(t)$ can be understood as an instantaneous toughness taking into account interactions between local toughness values through the dependence in the crack front geometry. As shown below, the crack propagation can be compared to a phase transition from the pinned state to an unpinned (i.e., propagation) regime, where the loading plays the role of the control parameter. As usually observed in such second-order phase transition, the behavior close to $\kappa^{*}$ can be characterized by a series of critical exponents which are universal, i.e., independent of the local disorder introduced in the model, such as the distribution $p\left(K_{c}\right)$ of local toughness (see (Sornette, 2000) for a presentation of the background and a review of other occurrences of criticality in a variety of applications including mechanical and geophysical examples).

\section{Statistical properties of the steady state}

After a transient regime influenced by the initial crack geometry, the model gives rise to a steady state during which a number of statistical observables can be defined and characterized. The main properties of this steady state are recalled. More details can be found in (Tanguv et al., 1998; Skoe et al., 2002). 


\subsection{Front roughness}

The first interesting observation concerns the crack front morphology. No specific scale of roughness is evidenced between the discretization scale and the system size, but rather a scale invariant geometry appears. The crack front is observed to be a self-affine object such that the r.m.s. height difference between two points distant of $\Delta x$ scales as

$$
\left\langle[h(x+\Delta x)-h(x)]^{2}\right\rangle_{x}^{1 / 2} \propto \Delta x^{\zeta},
$$

with $\zeta \approx 0.37$, irrespective of the disorder. The same property can be analyzed with different tools (Schmittbuhl et al., 1995b), such as Fourier power spectra, wavelet analysis, probability density function (p.d.f.) of height differences, all revealing the same property.

\subsection{Activity}

The spatio-temporal distribution of activity [i.e., map of $x_{a c t}(t)$ vs. $t$ ] displays a highly non-trivial organization, without any characteristic scale, neither in space nor in time. More precisely, by studying the p.d.f. of the difference $d=\left|x_{a c t}(t+\Delta t)-x_{a c t}(t)\right|$ at a fixed time interval, two regimes are found:

- at a small distance, the p.d.f. is uniform;

- at large distances, the p.d.f. decays as a power-law $d^{-2}$ that reflects the kernel of the interaction term in Eq. (1).

The cross-over distance between these two regimes, $d^{*}$ depends on the time interval $\Delta t$ as a power-law

$$
d^{*} \propto \Delta t^{1 / z},
$$


where $z$ is the so-called dynamic exponent, related to the roughness exponent $\zeta$ by

$$
z=1+\zeta
$$

Such a behavior can be recovered in a class of stochastic processes, i.e., Linear Fractional Stable Motion (Krishnamurthy et al., 2000) that contains both a wide distribution of elementary $d$, and long-range temporal correlations.

The above property reveals that the propagation consists in localized bursts of activity, where the clustering of activity in space and time can be decomposed hierarchically from the larger ones down to the smaller ones in a scale-invariant fashion.

\subsection{Distribution of effective toughness}

The two above properties dealt with geometric organization that may be difficult to access experimentally. However, the model also provides access to the loading, and hence the effective macroscopic toughness either at each instant of time $\kappa(t)$ or globally $\kappa^{*}$. The interesting feature is that the signal $\kappa(t)$ contains some information about the spatio-temporal organization of activity.

The first observation is the statistical distribution of $\kappa(t)$ which shows a long tail of low effective toughness, but an abrupt fall-off of the distribution near the maximum value $\kappa^{*}$. The latter can be characterized to behave singularly as

$$
P(\kappa) \propto\left(\kappa^{*}-\kappa\right)^{\beta}
$$

irrespective of the microscopic toughness distribution $p\left(K_{c}\right)$. Figure 2 shows the distributions $P(\kappa)$ for two different distributions $p\left(K_{c}\right)$, either uniform in $[0 ; 1]$ or centered reduced Gaussian. 
To make the connection with the activity map, it is instructive to study the distribution of effective toughness $P(\kappa(t), d)$ conditioned by a jump between the activity site $\left|x_{a c t}(t+1)-x_{a c t}(t)\right|=d$. The clustered nature of the activity suggests that one needs to reach a locally strongly pinned configuration to observe a large distance jump of the active site. Thus the distribution $P(\kappa(t), d)$ is expected to concentrate progressively closer to $\kappa^{*}$ as $d$ increases (Skoe et al., 2002). This is what is observed in Fig. 3. Moreover, as $d$ becomes large compared to the discretization scale, it can be seen that the distribution $P(\kappa, d)$ converges to a unique form up to a scaling factor. Hence, one can write

$$
P(\kappa, d)=d^{\alpha} \psi\left[\left(\kappa^{*}-\kappa\right) d^{\alpha}\right]
$$

so that a characteristic correlation length $\ell_{\|}$can be derived for each loading $\ell_{\|} \propto\left(\kappa^{*}-\kappa\right)^{-\nu}$ where $\nu=1 / \alpha$. The physical meaning of such a length scale is that for a subcritical loading $k_{0}=\kappa$, starting from any configuration in the steady state, the activity is localized in a region of extent $\ell_{\|}$. The time needed to reach a stable configuration can be obtained from the previous scaling properties, $\Delta t \propto \ell_{\|}^{z} \propto\left(\kappa^{*}-\kappa\right)^{-\nu z}$. It can be demonstrated (Fisher, 1985; Kardar, 1998) that

$$
\nu=\frac{1}{1-\zeta},
$$

or this scaling property can be read off from the interaction kernel in Eq. (1).

Knowing the statistics of jumps $d$ distributed as a power-law of exponent -2 , together with the scaling properties of the conditioned distribution $P(\kappa, d)$, allows one to compute the overall distribution of $\kappa$ in the vicinity of $\kappa^{*}$. It provides the critical behavior described by Eq. (9) with

$$
\beta=\nu-1=\frac{\zeta}{1-\zeta}
$$

Table 1 recalls the theoretical expressions of all the critical exponents in- 
troduced so far, together with their numerical value. All exponents can be expressed as a function of one of them $(e . g ., \zeta)$. However, the numerical value of the latter is not known theoretically. Numerical simulations are needed to obtain an accurate estimate. Functional renormalization group methods have been used (Nattermann et al., 1993; Naravan and Fisher, 1993) to provide an analytic route to estimate $\zeta$. However, it remains a perturbative method, which even when computed up to second order (Le Doussal et al., 2002), remains approximative.

\section{Crack arrest}

The discussion has been focused here on the steady state propagation in a homogeneous environment. With those results, the problem of crack arrest can be addressed in a geometry where the stress intensity factor decreases with the propagation distance.

In (Chudnovskv and Kunin, 1987; Jeulin, 1994; Charles and Hild, 2002; Charles et al., 2003), the problem is treated by using a simple one dimensional description. Only the mean propagation distance $\langle h\rangle_{x}$ is used to parameterize the crack advance. Accordingly, the stress intensity profile, $K\left(\langle h\rangle_{x}\right)$, is introduced, together with some effective toughness $K_{c}\left(\langle h\rangle_{x}\right)$. At this level of description, the effective toughness is assumed to be given by a random function, with a correlation length representative of the microstructure. Thus this random function is modeled as a piecewise constant function over a "grain size" $1 / \lambda$, without any correlation above this scale. The grain size is either constant (Charles and Hild, 2002; Charles et al., 2003) or described by using a Poisson mosaic (Jeulin, 1994). The p.d.f. for the effective toughness, $f\left(K_{c}\right)$ 
is introduced.

Let us briefly recall the argument developed in (Charles and Hild, 2002; Charles et al., 2003). Let $F(K)=P\left(K_{c}<K\right)$ be the cumulative probability of effective toughness $F(K)=\int_{0}^{K} f\left(K_{c}\right) \mathrm{d} K_{c}$. The probability $Q(h)$ that the crack arrest distance be larger than $h$ is then computed by writing that the condition $K_{c}(y)<K(y)$ hold for all $y \leq h$ (i.e., a "strongest" link assumption)

$$
Q(h)=\prod_{i=1}^{h \lambda} F(K(i / \lambda))
$$

with

$$
K(i / \lambda)=\min _{y \in[(i-1) / \lambda, i / \lambda[} K(y) .
$$

The continuous approximation of Eq. (13)

$$
Q(h) \approx \exp \left(\lambda \int_{0}^{h} \log [F(K(y))] \mathrm{d} y\right)
$$

holds for long distances as compared to the "grain size" $h \lambda \gg 1$ and a smooth stress intensity factor profile $\lambda d K(y) / d y \ll K(y)$. This simple result was then extended to the more complex case of subcritical crack growth (Charles et al., 2003). However, this situation is not considered in the present study.

Let us note that a similar expression can be found by assuming that the grain boundaries are defined by a Poisson tessellation (Jeulin, 1994). Under this assumption, $1 / \lambda$ is the average grain size and the propagation probability with a decaying stress intensity factor can be written as (Jeulin, 1994; Charles and Hild, 2002)

$$
Q(h)=\exp \left(-\lambda \int_{0}^{h}[1-F(K(y))] \mathrm{d} y\right) .
$$

The key question that underlies this description is the identification of the physical meaning of the "grain size," and the effective toughness. In this con- 
text, the first sections that considered the roughening of the crack front are helpful to quantify more precisely these terms, and thus the domain of validity of this description. One of the major advantages of resorting to this propagation model is that a well-defined effective toughness is now available, naturally parameterized in "time" $t$, which is proportional to the mean crack advance $t=\langle h\rangle_{x} L /\left(\xi_{\|} \xi_{\perp}\right)$ where $\xi_{\perp}$ is the extension of an elementary crack advance during one time step (i.e., the rescaling of the length $L$ by $\xi_{\|}$is used to obtain the number of microstructural units).

As noted above, the crack front develops a self-affine roughness, thus the width of the front $w=\left(\left\langle h^{2}\right\rangle_{x}-\langle h\rangle_{x}^{2}\right)^{1 / 2}$ is dependent on the crack length $L$, obeying $w \propto \xi_{\perp}\left(L / \xi_{\|}\right)^{\zeta}$. Moreover, complex correlations in the effective toughness are present. However, those correlations only occur at time intervals smaller than the time $T$ needed to depin the crack front over its entire length. This defines the larger time scale for those correlations scaling as $T \propto\left(L / \xi_{\|}\right)^{z}$. Taking into account the fact that $L$ is present in the relationship between time and mean crack advance, the correlation length $1 / \lambda$ scales as

$$
1 / \lambda \propto \xi_{\perp}\left(L / \xi_{\|}\right)^{z-1} \propto \xi_{\perp}\left(L / \xi_{\|}\right)^{\zeta}
$$

Equivalently, the "grain size" $1 / \lambda$ is physically given by the width of the crack front. This signals a first non trivial feature, unexpected from the onedimensional picture. The "grain size," which has to be used, depends on the crack front length.

One arrives now at the most interesting output of the planar crack modeling: namely, the distribution of effective toughness. The power-law distribution of the effective toughness, which can be seen in the vicinity of $\kappa^{*}$, has already been discussed in Section 4.3. However, this power-law regime results from the 
collective interaction of the crack front motion at different scales. Hence such a distribution holds in a domain where strong correlations are present, and therefore it cannot be used in the proposed approach given in Eqs. (13) and (15) because of the necessary assumption of statistical independence. However, the scale (i.e., "grain size") at which the latter condition holds is known. At such a scale, one has to consider the maximum effective toughness over $1 / \lambda$. Such a quantity has already been used in the study of the effective toughness conditioned by the distance between consecutive active sites, $d$. It suffices to consider the largest distance $d=L / 2$ admissible to focus on the largest effective toughness. Moreover, the distribution of effective toughness converges for large $d$ to a universal scaling form given in Eq. (10). Thus the shape of the effective toughness distribution $f$ is given by the universal function $\psi$. It simply has to be translated to match $\kappa^{*}$ and scaled so that its standard deviation scales as $\left(L / \xi_{\|}\right)^{-1 / \nu}$. This is an important conclusion: When the crack length is large compared to the heterogeneity of the microstructure, the effective toughness assumes a unique universal form independent of the microstructure. However the standard deviation of the distribution is scale-dependent.

Figure 4 shows the shape of the universal distribution $\psi$. This function has a power-law behavior close to the origin, with the same exponent as the one of the global toughness distribution, i.e., $\psi(x) \propto x^{\beta}$. This implies that the effective toughness does not exceed the threshold $\kappa^{*}$. For large arguments $x, \psi(x)$ has a rapid decay to 0 . This behavior concerns the small effective toughness and turns out to be inessential as explicitly shown in the following section. 


\section{Application to indentation cracks}

\subsection{Propagation probability}

A natural application of the above discussion concerns indentation cracks. This situation is a typical case where the stress intensity factor decreases strongly with the distance $c$ from the indentation center, $K \propto M c^{-3 / 2}$, where $M$ is the applied load (Ponton and Rawlings, 1989a,b), so that cracks are generally confined to the immediate vicinity of the indent. However, radial/median cracks

grow assuming approximately a circular or partly circular shape (Lawn, 1993). Hence the effective crack front length increases proportionally to $c$. This is a significant difference with respect to the previously treated case where $L$ was assumed to remain constant.

The change of the crack front length with $c$ is responsible for a continuous change of both the length parameter $1 / \lambda$ and the distribution of effective toughness. Coming back to a one dimensional image, the problem consists now of a crack propagating through a series of effective grains of increasing size, the toughness of each grain being drawn from a distribution that is itself grain size dependent. The generalization of Eq. (15) leads to

$$
Q(c) \approx \exp \left[\int_{0}^{c} \log \left(F_{r}(K(r))\right) \lambda(r) \mathrm{d} r\right]
$$

where the "grain size" $1 / \lambda$ now depends on the crack front length, $1 / \lambda=B c$, with $B=\pi$ for a semi-circular crack shape, $2 \pi$ for a circular one. The $B$ value is not specified in the sequel to account for any crack shape provided it remains self-similar at different stages of growth. Moreover, since the numerical prefactors in the scaling properties are not given, their values can be 
disregarded at this stage

$$
\frac{1}{\lambda(c)} \propto \xi_{\perp}\left(\frac{c}{\xi_{\|}}\right)^{\zeta}
$$

and the cumulative probability $F_{r}(K)$ of propagating through that grain derives from a distribution whose shape is universal (i.e., independent on the details of the toughness disorder at the microstructural scale, provided the latter is small compared with the crack front length) but whose width directly depends on the crack front length $L \propto c$

$$
\begin{aligned}
F_{c}(K) & =1-\int_{K}^{K^{*}} f(k, c) \mathrm{d} k \\
& =1-\int_{0}^{\frac{K^{*}-K}{K^{*}}}\left(\frac{c}{\xi_{\|}}\right)^{\alpha} \psi\left[q\left(\frac{c}{\xi_{\|}}\right)^{\alpha}\right] \mathrm{d} q .
\end{aligned}
$$

Note that for $K(c)>K^{*}$, the crack cannot be arrested so that $F_{c}(K)=1$. This leads to the introduction of a characteristic crack size $c^{*}$ such that $K\left(c^{*}\right)=K^{*}$ which is a lower bound on the crack size at arrest. For a large crack size as compared to the scale of heterogeneities, effective toughness values will always remain in the immediate vicinity of $K^{*}$ (i.e., $F$ close to unity) so that the power-law behavior of $\psi$ at the origin can be used to give a more precise description of $\log \left(F_{c}(K)\right)$

$$
\log \left(F_{c}(K)\right) \propto-\frac{c}{\xi_{\|}}\left(\frac{K^{*}-K}{K^{*}}\right)^{\frac{1}{1-\zeta}} .
$$

where the scaling relations summarized in Table 1 have been used. Gathering these expressions, Eq. (18) can be rewritten as

$$
Q(c) \approx \exp \left\{-A\left(c^{*}\right)^{2-\zeta} \int_{c^{*}}^{c}\left(\frac{r}{c^{*}}\right)^{1-\zeta}\left(\frac{K^{*}-K(r)}{K^{*}}\right)^{\frac{1}{1-\zeta}} \frac{\mathrm{d} r}{c^{*}}\right\}
$$

where $A$ is a prefactor whose dependence with respect to the material parameters $\xi$ is

$$
A \propto \frac{\xi_{\|}^{\zeta-1}}{\xi_{\perp}}
$$


A change of variable $x=c / c^{*}$ using the scaling of the stress intensity factor $K(c)=K^{*}\left(c^{*} / c\right)^{3 / 2}$ then leads to

$$
Q\left(x c^{*}\right) \approx \exp \left\{-A\left(c^{*}\right)^{2-\zeta} \int_{1}^{x} u^{1-\zeta}\left(1-u^{-3 / 2}\right)^{\frac{1}{1-\zeta}} \mathrm{d} u\right\}
$$

The integral in Eq. (24) can be recast in terms of an incomplete beta function. It can be noted that the same distribution was chosen a priori in (Charles and Hild, 2002). Equation (24) therefore constitutes an a posteriori validation, even though the "grain size" is no longer constant in the present analysis.

If the further hypothesis $(u-1) \ll 1$ can be made (i.e., small standard deviation of the crack arrest distance as compared to $c^{*}$, in practice $x-1<$ $5 \%$, the following algebraic expression of the decay of $Q\left(x c^{*}\right)$ with $x$ results

$$
Q\left(x c^{*}\right) \approx \exp \left(-A^{\prime}\left(c^{*}\right)^{2-\zeta}(x-1)^{\frac{2-\zeta}{1-\zeta}}\right)
$$

where the numerical value of the above exponents is $(2-\zeta) /(1-\zeta) \approx 2.6$ and $2-\zeta \approx 1.63$, and the dependence of $A^{\prime}$ on the characteristic parameters $\xi_{\perp}$ and $\xi_{\|}$is similar to that of $A$ [Eq. (23)]. In particular, the width $\Delta$ of the distribution of reduced crack size $c / c^{*}$ appears to scale as $\Delta \propto\left(c^{*}\right)^{-(1-\zeta)} \approx\left(c^{*}\right)^{-0.63}$, becoming more and more deterministic as the load is increased, i.e., $\Delta \propto M^{-2(1-\zeta) / 3} \propto M^{-0.42}$, with a power-law behavior reminiscent of a Weibull-type result, but with the great difference that the exponent is independent of the concerned material!

\subsection{Analysis of indentation experiments}

The previous results are now applied to experimental data. Several indentation tests, with different applied masses $m$ (i.e., $M \propto m$ ), have been per- 
formed on an alumina $\left(\mathrm{Al}_{2} \mathrm{O}_{3}\right)$ ceramic. Figure 5 shows an SEM picture of

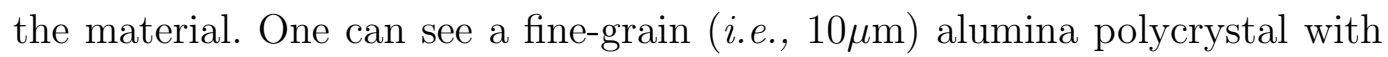
an intergranular glassy phase. A microanalysis shows that the latter contains $\mathrm{SiO}_{2}, \mathrm{CaO}$ and $\mathrm{Al}_{2} \mathrm{O}_{3}$ components. Vickers indentation with four different masses have been performed on previously polished sample surfaces: namely, $m=0.2 \mathrm{~kg}, 0.3 \mathrm{~kg}, 0.5 \mathrm{~kg}$ and $1 \mathrm{~kg}$. For each applied mass, mean indentation-generated crack lengths are measured. For the chosen loading range, it was checked that radial/median crack system is predominant by SEM observations and that $c / 2 a>1$, where $a$ is the size of the mean indent half-diagonal length (Ponton and Rawlings, 1989a, b). For each applied mass, measured crack lengths $c_{i}$ are associated to an experimental propagation probability $Q\left(c_{i}\right)$. The crack lengths are ranked in ascending order (i.e., $\left.c_{1}<c_{2}<\ldots<c_{N}\right)$ and the corresponding experimental probability are evaluated as $Q\left(c_{i}\right)=1-i /(N+1)$, where $N$ is the number of measured crack radii for the considered applied mass.

Equation (24) is used in the following analysis. Only two parameters have to be identified, namely the scale parameter $A$ and the characteristic radius $c^{*}$. Only one identification procedure is presented here, although a number of alternative methods can be used (Charles et al., 2003). Following Equation (24), for each load series, the best $c^{*}$ parameter is sought by prescribing that the constant $A$ be an intrinsic material parameter (i.e., unknown but identical for all series). Figure 6 shows the result of the identification. A good agreement is obtained. Table 2 gives the value of the parameters $c^{*}$ for the different applied masses. The value of $A^{1 /(2-\zeta)}$ is found to be equal to $0.15 \mu \mathrm{m}^{-1}$.

Furthermore, a rescaling procedure can be followed to compare all the experimental data on a single curve. Because of the algebraic expression of $Q$, one 
has to refer to a common conventional $c^{*}$, denoted as $c_{0}$, to be able to compare all data on a unique scale. Thus a rescaled propagation probability, $Q_{\text {rescaled }}$, is introduced such that

$$
Q_{\text {rescaled }}=Q^{q}
$$

where

$$
q=\left(\frac{c_{0}}{c^{*}}\right)^{2-\zeta}
$$

A check of the proposed scaling result is that $Q_{\text {rescaled }}$ plotted as a function of $c / c^{*}$ should fall onto a unique master curve. This is expected to be true for any $c_{0}$ choice. To treat all experimental points on an equal footing, the selection of the geometrical average of $c^{*}$ over all series was made, $c_{0}=\exp \left(\left\langle\log \left(c_{i}^{*}\right)\right\rangle\right)$ where $\langle\ldots\rangle$ denotes the arithmetic average. Figure 7 shows the prediction for the indentation experiments on alumina, and a good data collapse is observed.

Finally, Equation (24) also predicts that $c^{*}$ follows a power-law of the applied load $m$. Figure 8 shows this comparison in a log-log scale together with a line showing the expected power-law dependence (i.e., $c^{*} \propto m^{2 / 3}$ through the definition of a stress intensity factor $\left.K^{*} \propto m /\left(r^{*}\right)^{3 / 2}\right)$. Again a good agreement is obtained, although no more fitting parameter was used in this last confrontation.

\section{Conclusions}

A model for the propagation of a planar crack in a two-dimensional random landscape of toughness has been introduced. The complex interplay between the disorder and the elastic crack does not allow one to consider the successive failures of individual grains as independent events. The onset of propagation can be described as a genuine second order phase transition characterized by 
universal critical exponents.

When the toughness fluctuations are large enough or when the associated correlation length in the direction of propagation is short enough, the advance of the front stops being steady. The motion becomes jerky, the front jumps from one stable position to another one. In these strong pinning conditions the effective toughness of the material cannot be estimated by a simple arithmetic average of the toughness of the individual grains. These successive bursts take place over a continuous range of scales without any characteristic length besides the grain size and the lateral extension $L$ of the front. Over these scales the front develops universal features that do not depend on the details of the toughness disorder. In particular, the front roughness appears to be self-affine: its width scales as $w(L) \propto L^{\zeta}$ with $\zeta \approx 0.37$. The motion of the front is thus correlated up to the system size $L$ in the lateral direction and $L^{\zeta}$ in the direction of propagation. When a front of finite extent $L$ is considered, a coarse-grained approach at the scale of effective grains of size $L \times L^{\zeta}$ can be performed. A key result is that up to scaling factors only dependent on the system size, the distribution of effective toughness of these effective grains adopts a universal form. The latter is completely independent of the details of the microscopic toughness. If the lateral extent of the front remains constant during the propagation, the latter can thus be treated as the advance of a onedimensional crack through a series of effective grains characterized by the same well-defined effective toughness p.d.f. This result is extended to the case of selfsimilar cracks when the lateral front size is itself a growing quantity in the course of propagation. The coarse-grained approach remains valid but instead of advancing through effective grains of constant size, the one-dimensional crack propagates through effective grains of growing size together with a nonsteady toughness distribution. 
As an application to explore the predictive power of this scaling approach, the statistical distribution of crack arrest distances for indentation has been examined. The proposed scaling approach provides an analytical expression for the crack arrest length distribution whose dependence with respect to the material characteristics is limited to only two scalar parameters (a length scale, and a reference toughness). The rest of the analytical expression is universal, (independent of the considered material). These results have been validated against experimental data obtained from a polycrystalline sintered alumina.

\section{References}

J.-P. Bouchaud, E. Bouchaud, G. Lapasset and J. Planés, Models of fractal cracks, Phys. Rev. Lett. 71 (1993) 2240-2243.

E. Bouchaud, Scaling properties of cracks J. Phys. Cond. Mat. 9 [21] (1997) $4319-4344$

A. F. Bower and M. Ortiz, A Three-Dimensional Analysis of Crack Trapping and Bridging by Tough Particles, J. Mech. Phys. Solids 28 [5] (1991) 815858.

A. F. Bower and M. Ortiz, The Influence of Grain Size on the Toughness of Monolithic Ceramics, ASME J. Eng. Mat. Technol. 115 (1993) 228-236.

Y. Charles and F. Hild, Crack Arrest in ceramic/steel assemblies, Int. J. Fract. 15 [3] (2002) 251-272.

Y. Charles, F. Hild and S. Roux, Long-Term Reliability of Ceramics: the Issue of Crack Arrest, ASME J. Eng. Mat. Tech. 125 (2003) 333-340.

Y. Charles, F. Hild and S. Roux, work in progress, (2003).

A. Chudnovsky and B. Kunin, A Probabilistic Model of Brittle Crack Formation, J. Appl. Phys. 62 (1987) 4124-4129. 
W. A. Curtin, Toughening in Disordered Brittle Materials, Phys. Rev. B 55 (1997) 11270-11276.

W. A. Curtin, Stochastic Damage Evolution and Failure in Fiber-reinforced Composites, Adv. Appl. Mech. 36 (1998) 164.

A. G. Evans, Perspectives on the Development of High-Toughness Ceramics, J. Am. Ceram. Soc. 73 [2] (1990) 187-206.

D. S. Fisher, Sliding charge-density waves as a dynamic critical phenomenon, Phys. Rev. B 31 (1985) 1396-1427.

A. M. Freudenthal, Statistical Approach to Brittle Fracture, in: Fracture, H. Liebowitz, ed., (Academic Press, New York (USA), (1968) pp. 591-619).

H. Gao and J. R. Rice, A First Order Perturbation Analysis of Crack Trapping by Arrays of Obstacles, ASME J. Appl. Mech. 56 (1989) 828-836.

D. Jeulin, Fracture Statistics Models and Crack Propagation in Random Media, Appl. Mech. Rev. 47 [1] (1994) 141-150.

M. Kardar, Nonequilibrium dynamics of interfaces and lines, Phys. Rep. 301 (1998) 85-112.

S. Krishnamurthy, A. Tanguy, P. Abry and S. Roux, A stochastic description of extremal dynamics, Europhys. Lett. 51 (2000) 1-7.

B. R. Lawn, Fracture of Brittle Solids, (Cambridge University Press, Cambridge (UK), 1993).

P. Le Doussal, K. J. Wiese and P. Chauve, Two-loop functional renormalization group theory of the depinning transition, Phys. Rev. B 66 (2002) 174201-1-34.

O. Narayan and D. S. Fisher, Threshold critical dynamics of driven interfaces in random media, Phys. Rev. B 48 (1993) 7030-7042.

T. Nattermann, S. Stepanow, L.-H. Tang and H. Leschhorn, Dynamics of interface depinning in a disordered medium, J. Phys. II (France) 2 (1993) 
1483-1488.

C. B. Ponton and R. D. Rawlings, Vickers Indentation Fracture Toughness Test - Part 1 - Review of Literature and Formulation of Standardized Indentation Toughness Equations, Mat. Sci. Tech. 5 (1989) 865-872.

C. B. Ponton and R. D. Rawlings, Vickers Indentation Fracture Toughness Test - Part 2 - Application and Evaluation of Standardized Indentation Toughness Equations, Mat. Sci. Tech. 5 (1989) 961-976.

S. Ramanathan and D. S. Fisher, Onset of Propagation of Planar Cracks in Heterogeneous Media, Phys. Rev. B 58 (1998) 6026-6046.

S. Roux, D. Vandembroucq and F. Hild, Effective toughness of heterogeneous brittle materials, Eur. J. Mech. A/Solids [in press] (2003).

J. Schmittbuhl, S. Roux, J.-P. Vilotte and K. J. Måløy, Pinning of interfacial crack: Effect of non-local interactions, Phys. Rev. Lett. 74 (1995) 1787-1790.

J. Schmittbuhl, J.-P. Vilotte and S. Roux, Reliability of self-affine measurements, Phys. Rev. E 51 (1995) 131-147.

R. Skoe, D. Vandembroucq and S. Roux, Front Propagation in Random Media: From Extremal to Activated Dynamics, Int. J. Modern Physics C 13 (2002) 751-757.

D. Sornette, Critical phenomena in Natural Sciences, (Springer Verlag, Berlin (Germany), 2000).

A. Tanguy, M. Gounelle and S. Roux, From individual to collective pinning: Effect of long-range elastic interactions, Phys. Rev. E 58 (1998) 1577-1590. W. Weibull, A Statistical Theory of the Strength of Materials, (Roy. Swed. Inst. Eng. Res., Report 151, 1939). 


\section{List of Tables}

1 Summarv of the theoretical expressions and numerical values of the critical exponents define
2 Values of the parameters $c^{*}$ for the four different applied masses $m$ when the parameter $A$ is 
Table 1

Summary of the theoretical expressions and numerical values of the critical exponents defined in the text expressed as a function of $\zeta$.

\begin{tabular}{|c|c|c|}
\hline Exponent & Expression & Value \\
\hline$\zeta$ & & 0.37 \\
$\nu$ & $1 /(1-\zeta)$ & 1.59 \\
$\alpha$ & $(1-\zeta)$ & 0.63 \\
$\beta$ & $\zeta /(1-\zeta)$ & 0.59 \\
$z$ & $(1+\zeta)$ & 1.37 \\
\hline
\end{tabular}

Charles et al. 
Table 2

Values of the parameters $c^{*}$ for the four different applied masses $m$ when the parameter $A$ is assumed to be load-independent.

\begin{tabular}{|c|c|}
\hline Mass $m(\mathrm{~kg})$ & $c^{*}(\mu \mathrm{m})$ \\
\hline 0.2 & 11 \\
0.3 & 14 \\
0.5 & 21 \\
1.0 & 36 \\
\hline
\end{tabular}

Charles et al. 


\section{List of Figures}

1 Depiction of crack propagation along the $y$-direction through a random toughness field show 2 Distribution of the effective toughness $\kappa(t)$ obtained for a uniform toughness distribution in

3 Distribution of the effective toughness $\kappa(t)$ obtained for a uniform toughness distribution in

4 Universal function $\psi(u)$ giving the distribution of the effective toughness. Note that the orig:

5 Alumina used in the studv with an average grain size of the order of $10 \mu \mathrm{m}$. One can note th

6 Propagation probability $Q$ versus crack radius $c$ for four different applied masses. The svmbc

7 Rescaled propagation probability $Q_{\text {recaled }}$ versus dimensionless crack radius $c / c^{*}$ for four diff

$8 \quad$ Parameter $c^{*}$ versus applied mass $m$. A power law with an exponent of $2 / 3$ fits reasonably w 


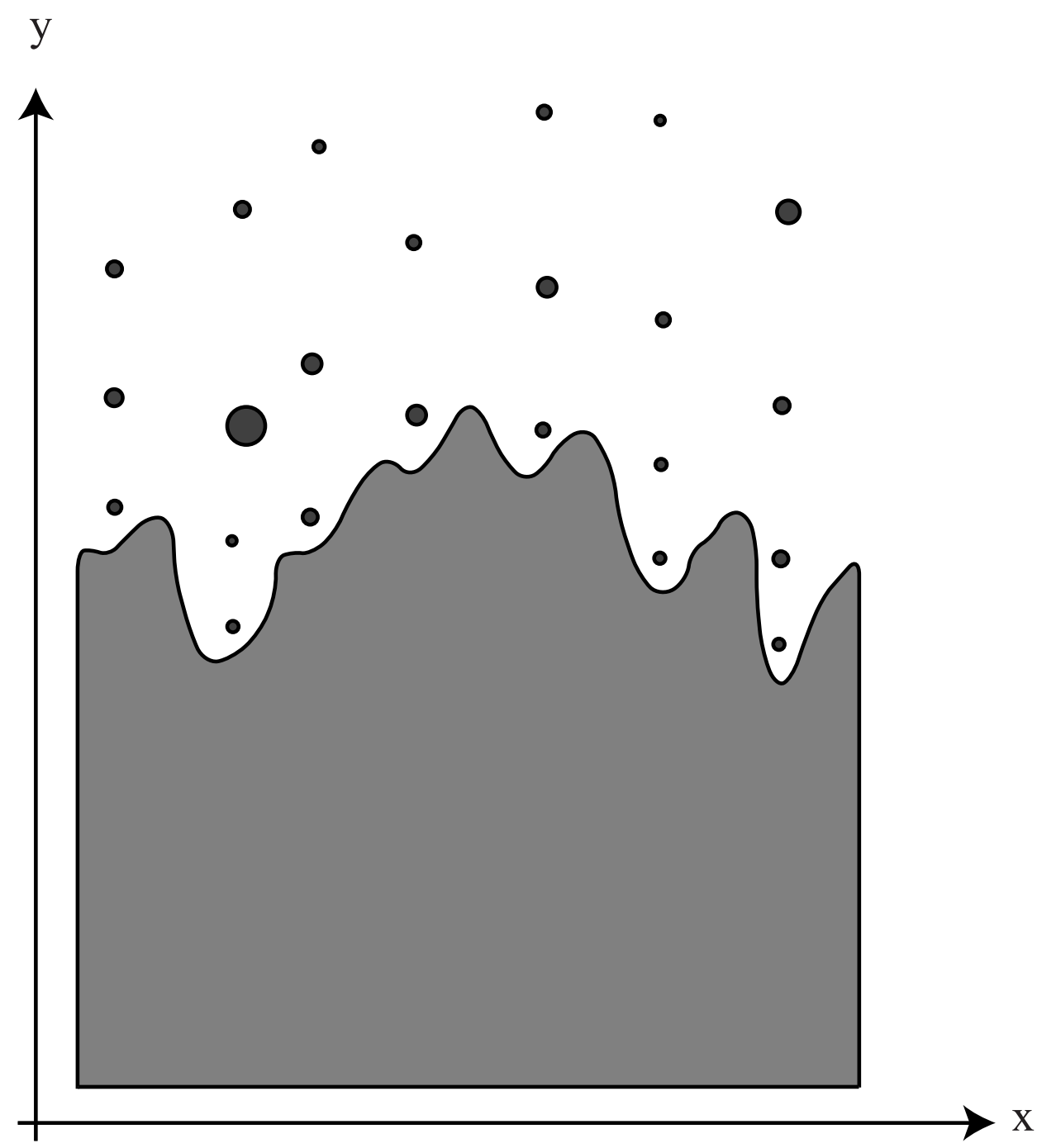

Fig. 1. Depiction of crack propagation along the $y$-direction through a random toughness field shown here as a collection of inclusions which may pin the crack front.

Charles et al. 


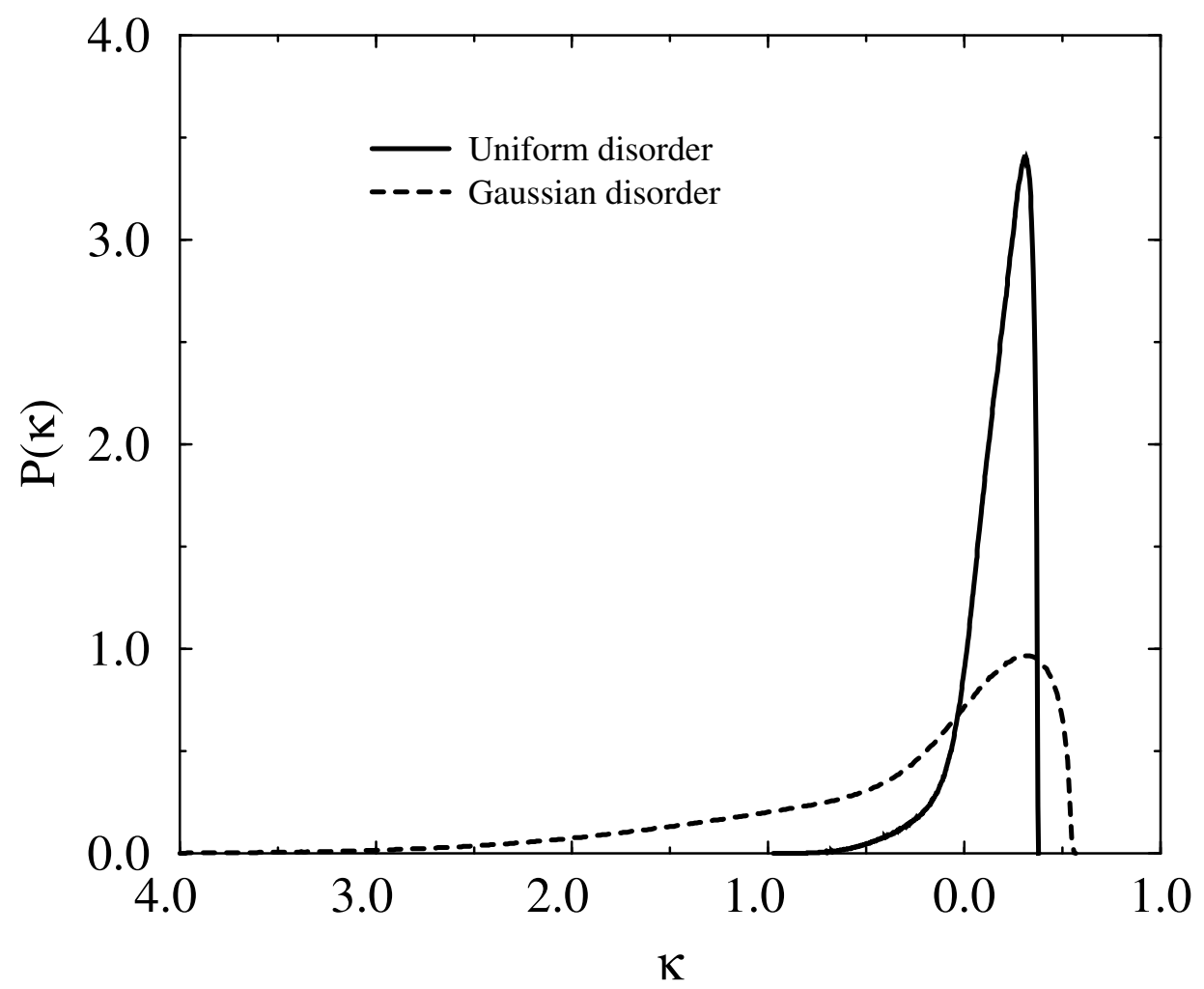

Fig. 2. Distribution of the effective toughness $\kappa(t)$ obtained for a uniform toughness distribution in [0;1] (solid line) and centered reduced Gaussian (dashed line).

Charles et al. 


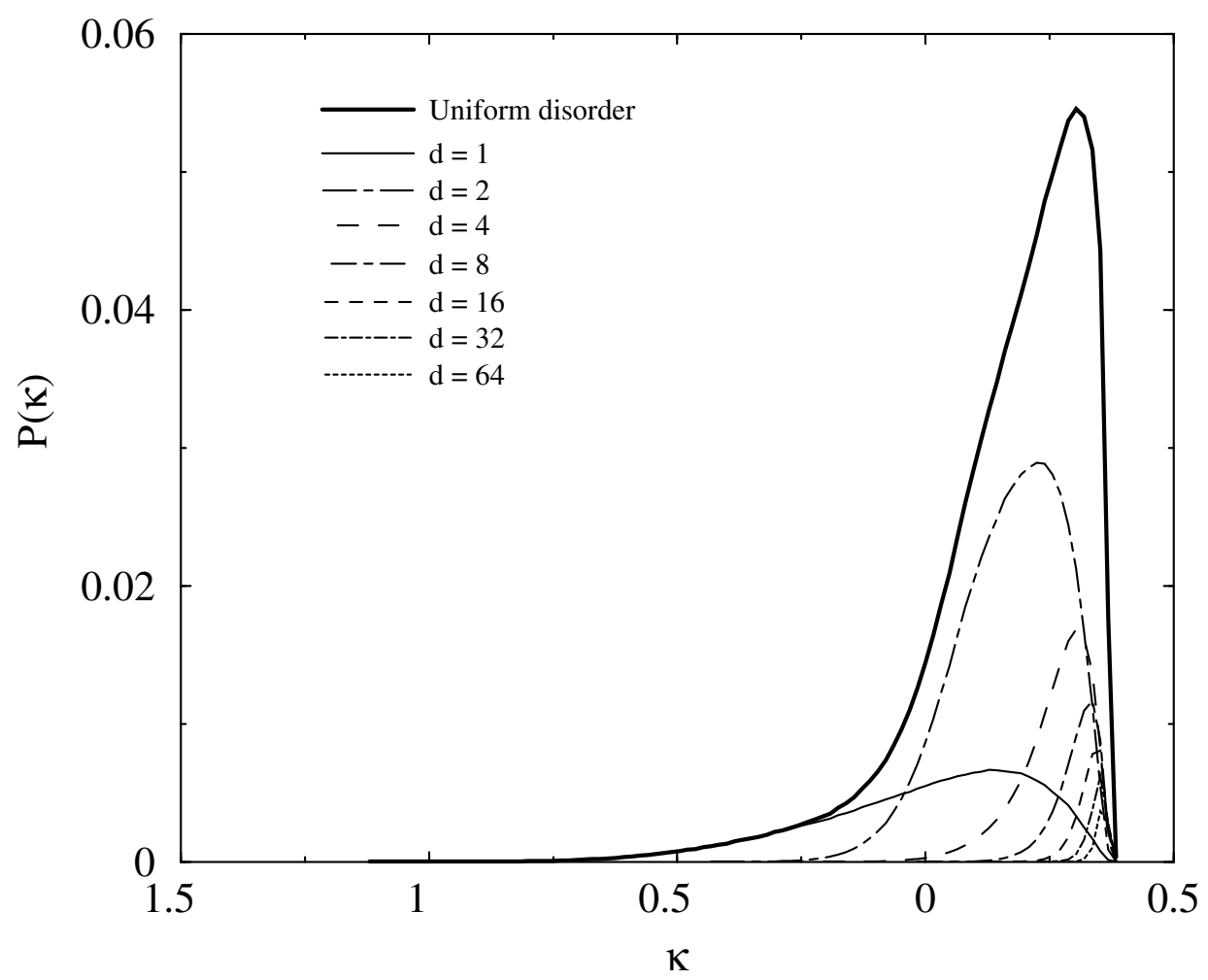

Fig. 3. Distribution of the effective toughness $\kappa(t)$ obtained for a uniform toughness distribution in $[0 ; 1]$, and conditioned distributions for geometrically distributed jump distances from $d=1$ to $d=64$ progressively concentrating close to $\kappa^{*}$. The conditioned distributions are not normalized so that they represent their contribution to the overall distribution $P(\kappa)$.

Charles et al. 


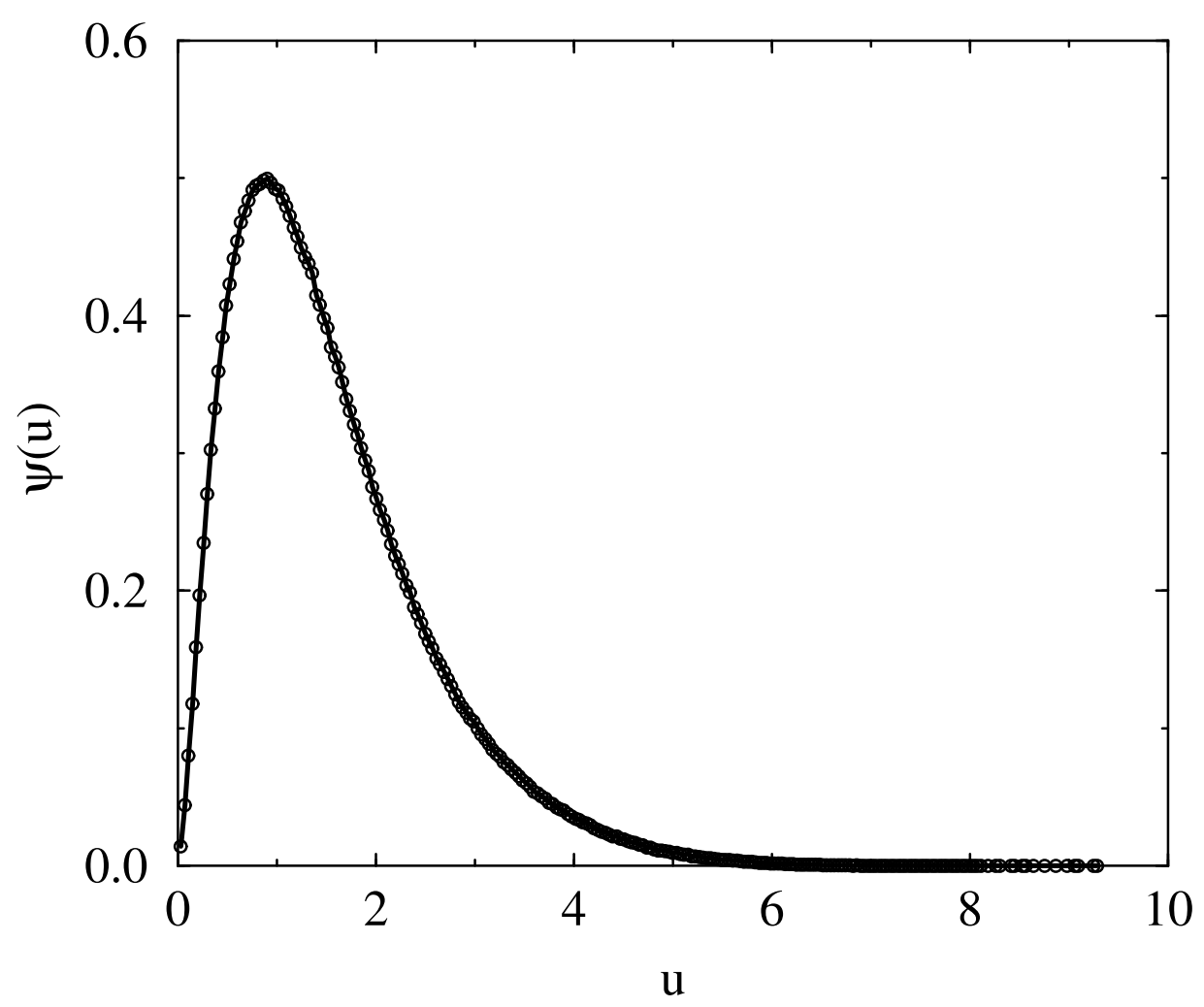

Fig. 4. Universal function $\psi(u)$ giving the distribution of the effective toughness. Note that the origin $u=0$ corresponds to $\kappa=\kappa^{*}$, and increasing $u$ correspond to decreasing $\kappa$ values.

Charles et al. 


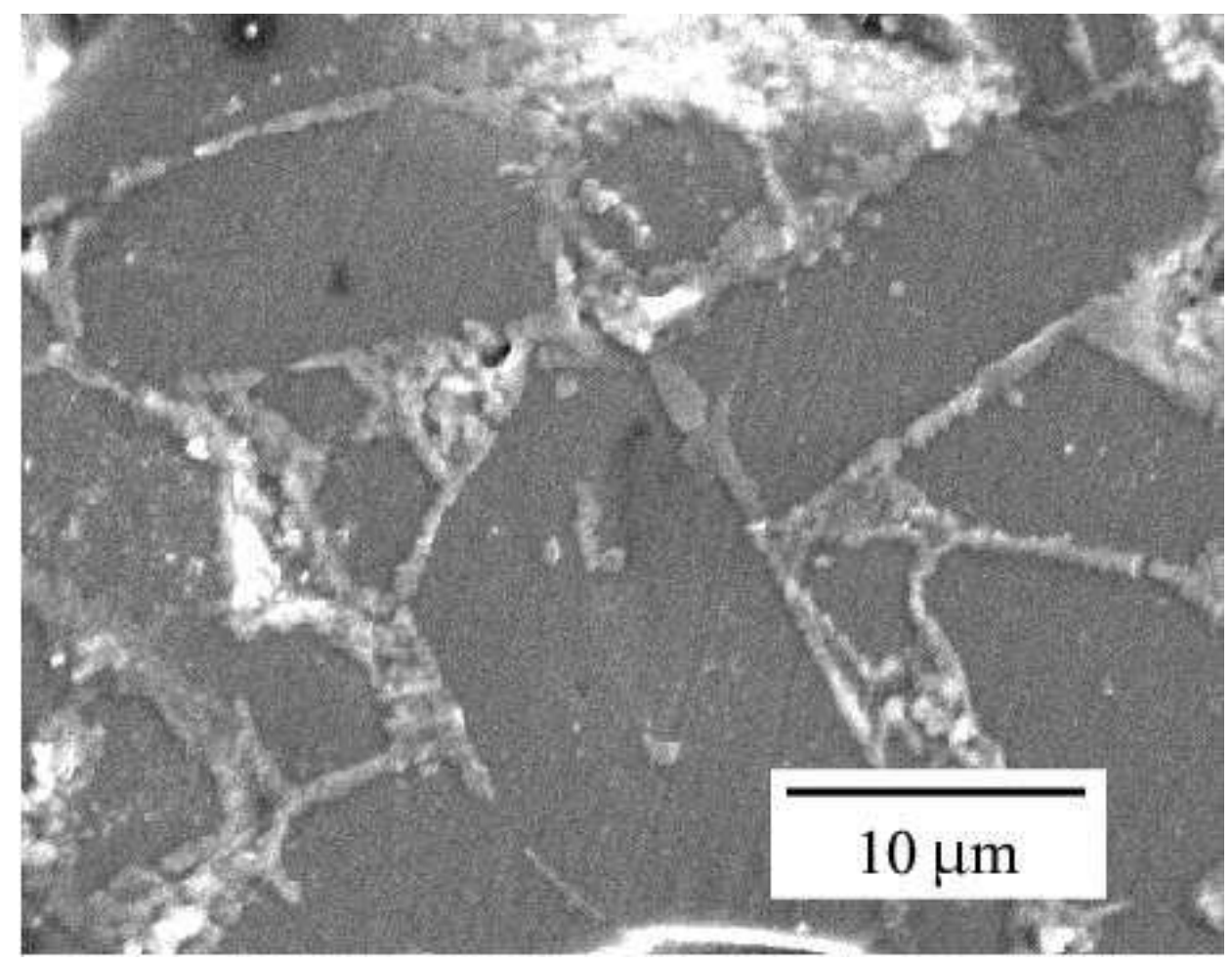

Fig. 5. Alumina used in the study with an average grain size of the order of $10 \mu \mathrm{m}$. One can note the glassy intergranular phase.

Charles et al. 


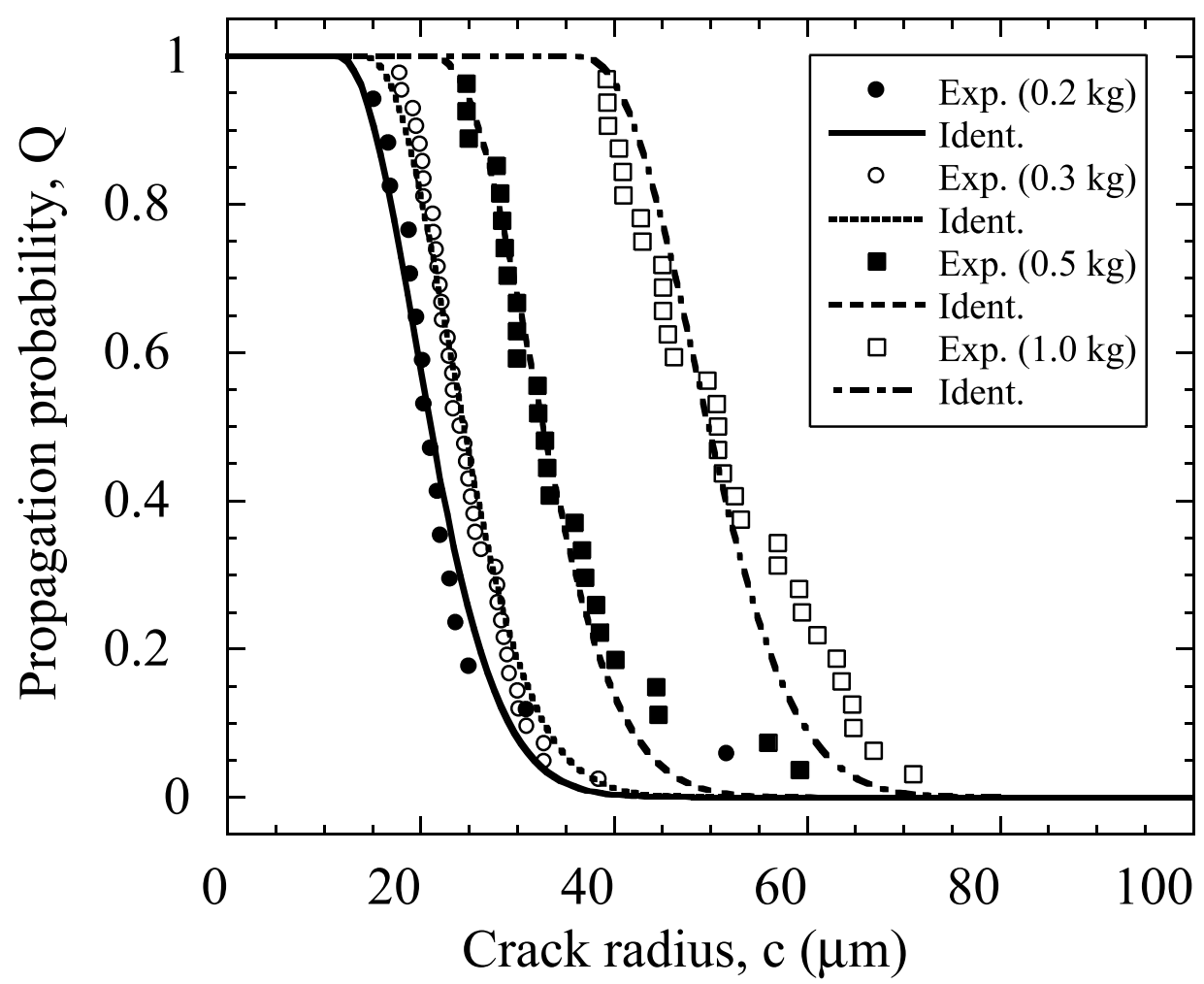

Fig. 6. Propagation probability $Q$ versus crack radius $c$ for four different applied masses. The symbols are experimental data and the lines are identifications when the parameter $A$ is assumed to be load-independent.

Charles et al. 


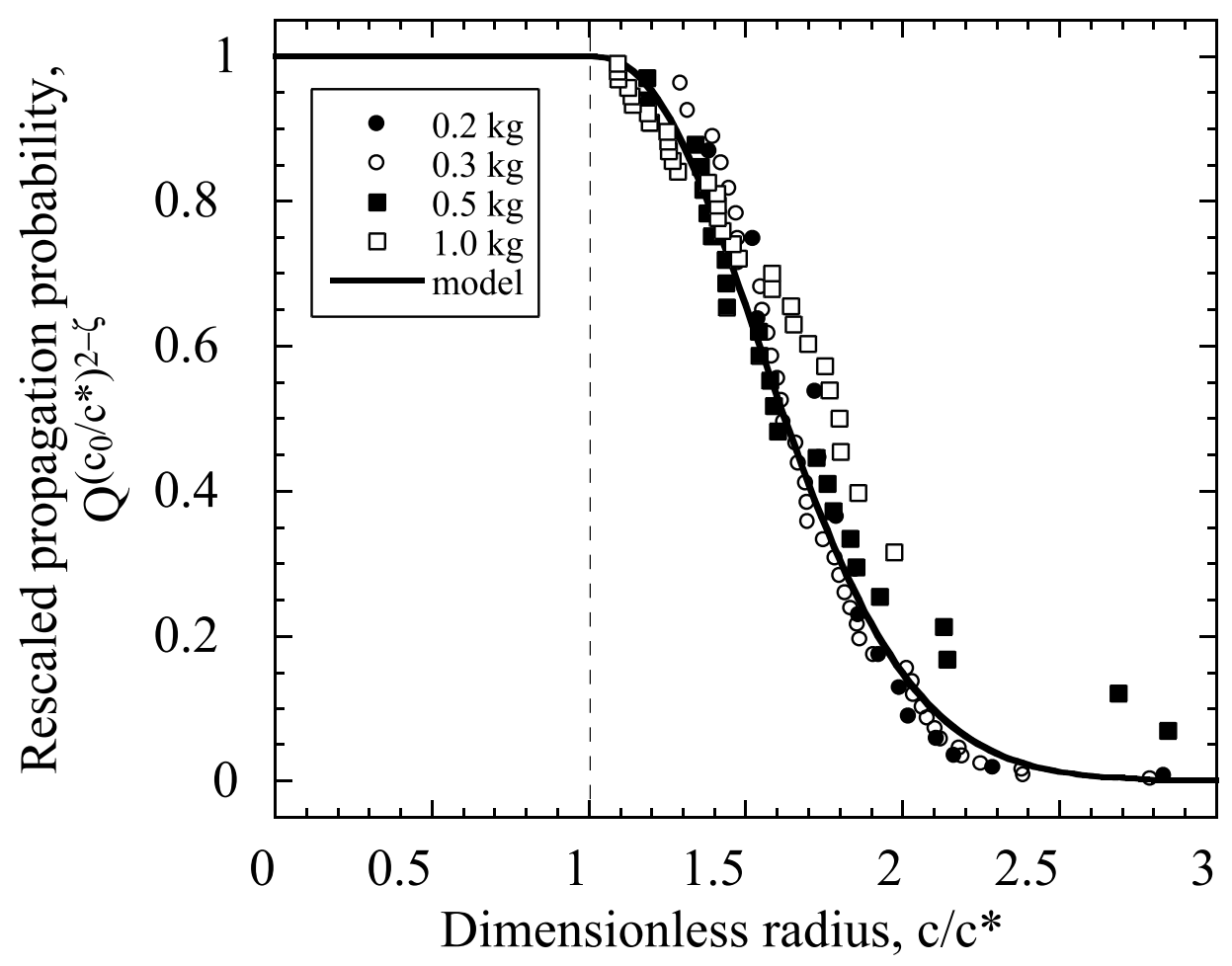

Fig. 7. Rescaled propagation probability $Q_{\text {rescaled }}$ versus dimensionless crack radius $c / c^{*}$ for four different applied masses. The symbols are experimental data and the curve is the result of the identification with a single parameter $A$. From the present analysis, it is expected that all experimental points should fall on the same curve.

Charles et al. 


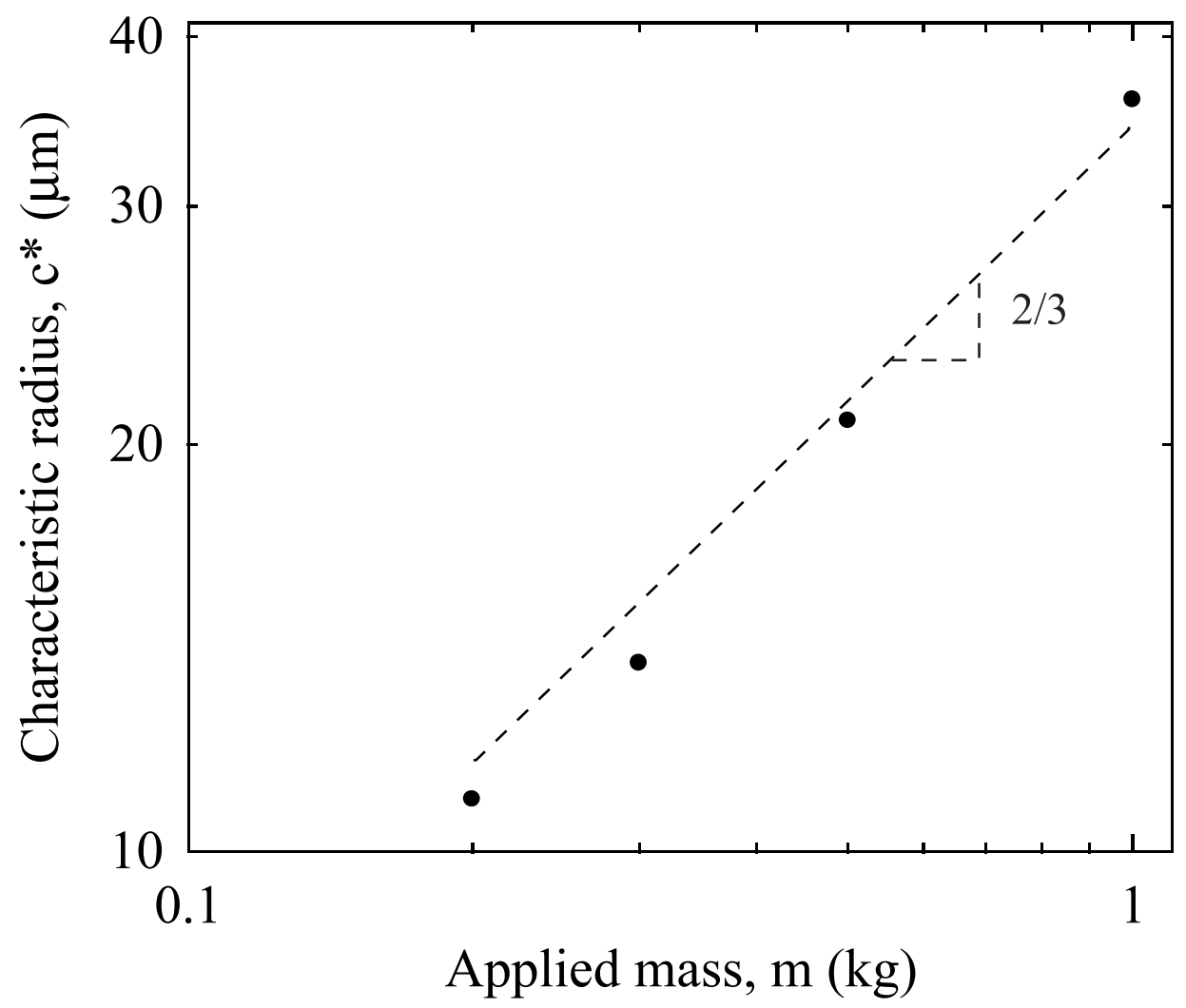

Fig. 8. Parameter $c^{*}$ versus applied mass $m$. A power law with an exponent of $2 / 3$ fits reasonably well the experiments (dashed line).

Charles et al. 